\title{
Analysis of Regional Economic Development Differences and Causes in Guangdong Province
}

\author{
Juntao Song \\ Shenzhen Experimental middle School, China. \\ oliviasong1026@outlook.com
}

\begin{abstract}
In this paper, ESDA and difference index are used to explore the spatial difference characteristics and evolution trend of the urban economy in Guangdong, and the main factors influencing regional economic difference in Guangdong are measured by using spatial error model. The conclusion shows that the urban economy of Guangdong province shows some differences in spatial distribution and tends to coordinate in time development. Regional policies are the main factors influencing economic differences, and fiscal expenditure, consumption, import and export and investment all affect economic differential development to different degrees. But the positive spatial overflow effect makes the economic difference in Guangdong province tend to converge in general.
\end{abstract}

Keywords: regional economy, influencing factor, spatial error model, overflow effect.

\section{Introduction}

Behind more than 30 years of rapid economic development in reform and opening-up, there are a series of imbalance in economy, society, resources, environment and culture. The "coordinated development" proposed in recent years is to solve the problem of imbalance in the development process [1]. As a leading demonstration area of reform and opening up, Guangdong leads the country in economic development, but its regional economic development is uneven. Although it has basically achieved the goal of "a few people get rich first", Guangdong still has a long way to go on the road of "finally achieving common prosperity". In recent years, Guangdong's per capita GDP growth rate has been running at a low level, showing signs of further decline [2]. In order to successfully cross the "middle-income trap", Guangdong needs to realize the transformation of economic development mode and achieve coordinated development in the short term.

Research on regional differences from the perspective of economic development is the mainstream research direction nowadays, and many scholars have conducted relevant research and analysis from different perspectives [3]. However, there are few empirical studies on regional economic differences in Guangdong, and few literatures delve into the causes of the differences. In this paper, from the perspective of empirical research, the economic Data of 21 cities in Guangdong province from 2003 to 2016 is selected and Exploratory Spatial Data Analysis (ESDA) [4], statistical analysis and spatial econometric analysis are used comprehensively to explore the distribution characteristics and development trend of regional economic Spatial differences in Guangdong. Based on the macroeconomic theory, this paper deduces the causes of economic differences and builds a panel data spatial error model to test the influencing factors and effects of regional economic differences in Guangdong.

\section{Research Methods}

(1) Exploratory spatial data analysis

Exploratory spatial data analysis (ESDA) is a research method that utilizes modern geographic information system to carry out mathematical statistics and surface demonstration of spatial unit attributes, in order to explore spatial distribution characteristics and mutual relations of geographical units. This paper mainly uses the global spatial autocorrelation (Global Moran's I) coefficient, which can reflect the correlation of spatial units, to study the degree of economic correlation between Guangdong cities. The calculation formula is: 


$$
I=\frac{N}{S_{0}} \times \frac{\sum_{i=1}^{n} \sum_{j=1}^{n} w_{i, j} z_{i} z_{j}}{\sum_{i=1}^{n} z_{i}^{2}}
$$

Where, $z_{i}$ is the deviation between the economic level of region $i$ and its average value. $w_{i, j}$ is the spatial weight between factor $i$ and $j$, with 1 as adjacent and 0 as non-adjacent. $N$ is the total number of regions, $S_{0}$ is the set of all spatial weights. Moran's I value is between -1.0 and 1.0. Moran's I $>0$ means that the economy of each city is positively correlated. The larger the value is, the more obvious the spatial correlation is. Moran's I $<0$ means that the economy of each city is negatively correlated, and the smaller the value, the greater the spatial difference.

(2) Spatial econometric analysis

It is a scientific and effective method to construct a spatial econometric model to analyse the cause of regional economic spatial differences. The factors affecting regional economy such as fixed asset investment is selected as explanatory variables, and per capita GDP is taken as explained variables. The spatial error model of panel data is used to build the spatial difference model of Guangdong regional economy, as shown in formula (2):

$$
\left\{\begin{array}{l}
y_{i t}=\alpha+X_{i t} \beta+u_{i t} \\
u_{i t}=\rho W u_{i t}+\varepsilon \\
\varepsilon \sim N\left(0, \sigma^{2}\right)
\end{array}\right.
$$

In model (2), $y_{i t}$ is the per capita GDP level of city $i$ in year $t, X_{i t}$ is the relevant explanatory variable of city $i$ in year $t$, and is the regression coefficient to be estimated. $u_{i t}$ is the error vector, $\varepsilon$ is the error coefficient, and is the spatial weight matrix. $p$ is the space error coefficient, which reflects the spatial interaction.

\section{Measurement of Regional Economic Spatial Differences in Guangdong}

\subsection{Index Selection and Basic Data Analysis}

In the selection of index, the scientific and objectivity of index such as total output value, material products, national disposable income, per capita GDP and consumer expenditure are comprehensively compared. According to the research conclusions of Liu xiaoming, Wei yingqi and Li guoping [5], per capita GDP is a relatively good index to measure regional economic difference in terms of the objectivity, expressiveness and recognition of index. Here, per capita GDP is selected as the index to measure the urban economic development level of Guangdong, so as to eliminate the impact of regional economic imbalance caused by the difference in population size. From 2003 to 2016, the per capita GDP level of Guangdong region was on the whole increasing, but in recent years, affected by the peripheral environment, its growth rate has showed signs of slowing down. According to the change trend of annual per capita GDP data of each city, it is found that the extreme difference of each city's annual economy on the basis of overall development is also increasing year by year, indicating that the data variability is increasing.

\subsection{Variation of ESDA Measured Spatial Differences}

Exploratory spatial data analysis (ESDA) is used to compare regional economic difference in Guangdong in different years, and standard deviation is selected as a grading index. The illustrations of per capita GDP in various cities of Guangdong in 2003 and 2016 are respectively marked. From dark to light, the colour represents the spatial distribution of cities with standard deviation above 1.5 per capita GDP, cities with standard deviation between 0.5 and 1.5 per capita GDP, cities with 
standard deviation between -0.5 and 0.5 and cities with standard deviation less than -0.5 , as shown in figure 1.

By comparing the spatial differences of per capita GDP in cities of Guangdong in 2003 and 2016 in figure 1, it can be found that the cities in the first tier of per capita GDP are Guangzhou, Shenzhen and Zhuhai. The cities of the second tier are increased from Foshan to Zhongshan. Yangjiang, Zhaoqing and Shaoguan are promoted from the fourth tier to the third tier. That reduced the number of fourth tier cities from 11 in 2003 to eight in 2016.
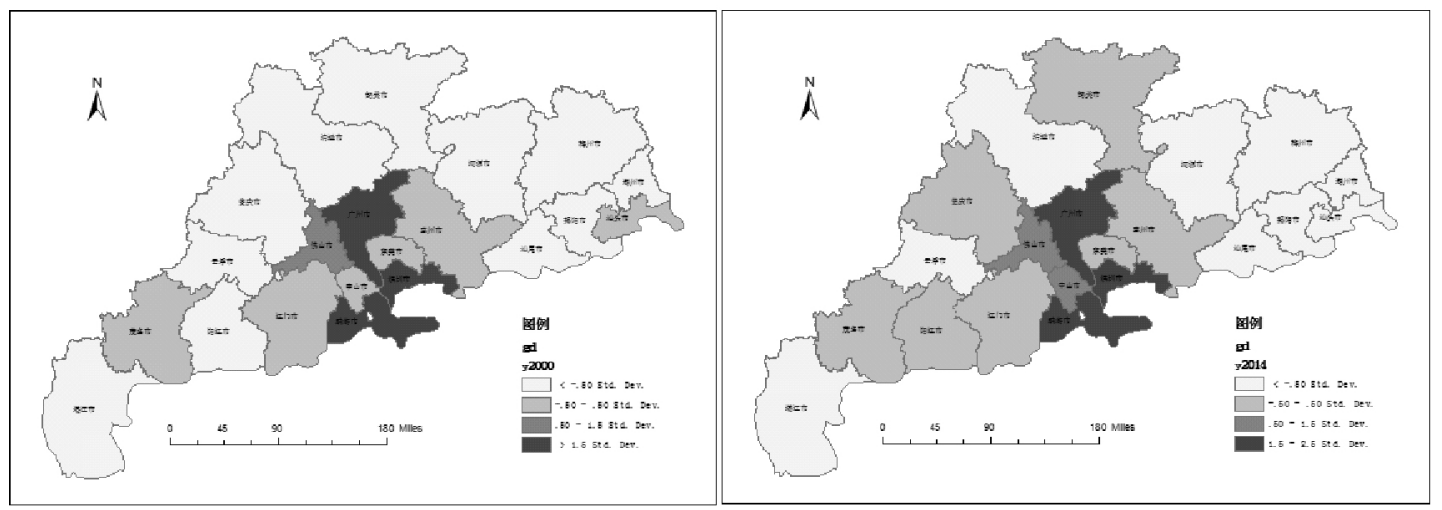

Spatial differences of per capita GDP in 2003 Spatial differences of per capita GDP in 2016

Figure 1. Comparison of spatial differences of per capita GDP in cities of Guangdong in 2003 and 2016

From exploratory spatial data analysis (ESDA), it is not difficult to find that there are obvious spatial differences in Guangdong urban economy. From the perspective of development trend, the past 14 years have witnessed a further economic development towards the core cities of the pearl river delta and a convergence development trend from the pearl river delta radiating to the surrounding cities. Judging from the overall economic development trend, relatively developed regions are increasing and backward regions are decreasing. Guangdong's overall economy tends toward coordinated development.

\subsection{Development Trend of Differences Measured by Statistical Index}

In order to more accurately explore the development trend of urban economic difference in Guangdong province on the timeline, economic difference statistical indexes are introduced to calculate the Gini coefficient, Theil index, variation coefficient and Herfindahl index of per capita GDP of 21 cities in Guangdong province from 2003 to 2016, as shown in figure 2.

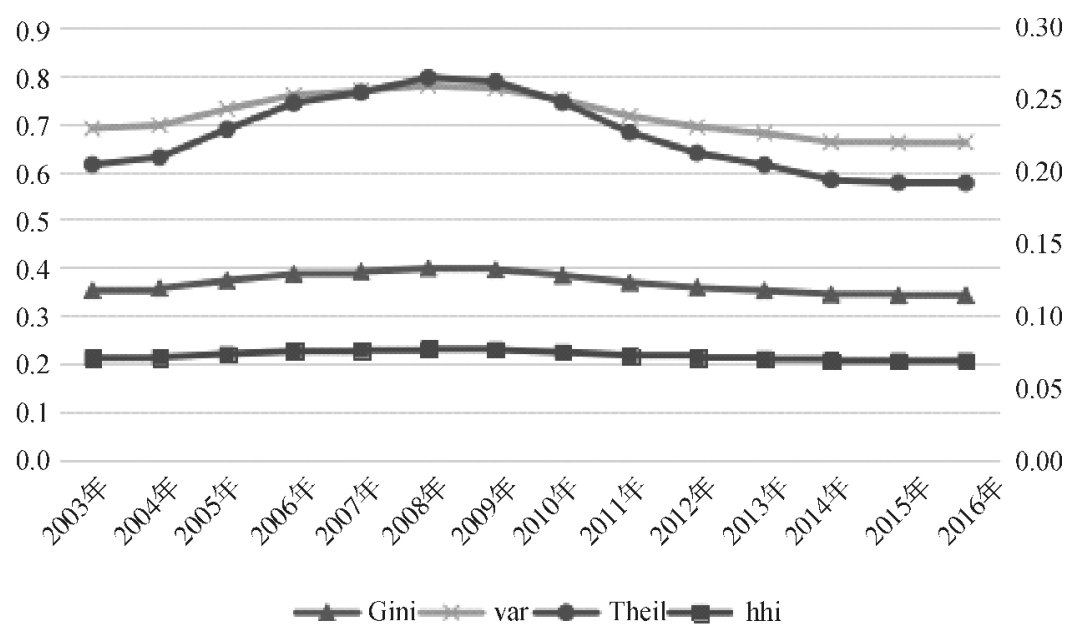

Figure 2. Trend chart of four indicators of economic difference 
As shown in figure 2, the overall evolution of regional economic difference index in Guangdong shows an inverted U-shape trend, among which the Theil index and the variation coefficient are the most obvious, indicating that the economic difference between Guangdong cities has first expanded and then narrowed since 2003. The inflection point occurred around 2008, and it shows a trend of continuous convergence in recent years, which further verifies that the regional economic difference in Guangdong has been shrinking in recent years, and also indicates that the regional economy in Guangdong is becoming more coordinated. According to Bernard's research conclusion, there is a certain correlation between economic growth and regional difference. Through comparison, it is found that the form of the four different indexes and the smooth trend of economic growth in Guangdong have the same track in the trend, indicating that in the period of the rapid development of Guangdong's overall economy, the regional differences are constantly expanding. When the overall economic growth slows down, regional differences gradually narrow.

Although from the overall level of Guangdong province, Guangdong regional economic difference has been shrinking in recent years. However, from the perspective of the four regions of pearl river delta, eastern Guangdong, western Guangdong and northern Guangdong, the regional economic difference in Guangdong is still expanding, as shown in figure 3 and 4.

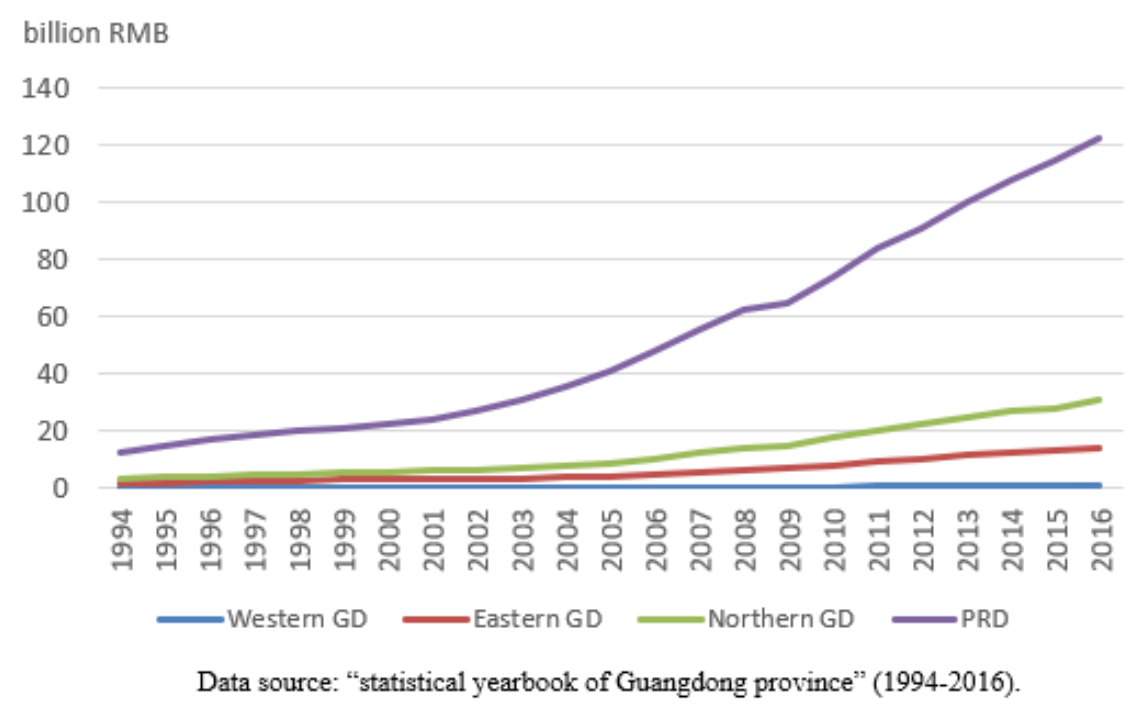

Figure 3. The GDP growth process of the four regions in Guangdong from 1994 to 2016 Data source: "statistical yearbook of Guangdong province" (1994-2016).

It can be seen from figure 3 that the GDP growth trend of the pearl river delta is significantly higher than that of eastern, western and northern Guangdong, and the economic difference between eastern, western and northern Guangdong is expanding. In 1994, the GDP of the pearl river delta region accounted for $74.2 \%$, while that of the eastern, western and northern Guangdong accounted for $9.16 \%, 9 \%$ and $7.59 \%$ respectively. In 2016, the GDP of the pearl river delta region increased to $81.1 \%$ in the whole province, while that of eastern Guangdong decreased to $4.77 \%$, western Guangdong to $7.8 \%$, and northern Guangdong to $6.37 \%$.

It can be seen from figure 4 that the per capita GDP growth trend of the pearl river delta is significantly higher than that of eastern, western and northern Guangdong, and the economic difference between eastern, western and northern Guangdong is expanding. In 1994, the GDP of the pearl river delta region accounted for $50.3 \%$ of the province, while the eastern, western and northern Guangdong accounted for $18.1 \%, 16.6 \%$ and $15 \%$ respectively. In 2016, the per capita GDP of the eastern Guangdong increased to $21 \%$ of the province, while that of the pearl river delta region dropped to $48.2 \%$, western Guangdong to $16 \%$, and northern Guangdong to $15.3 \%$. 


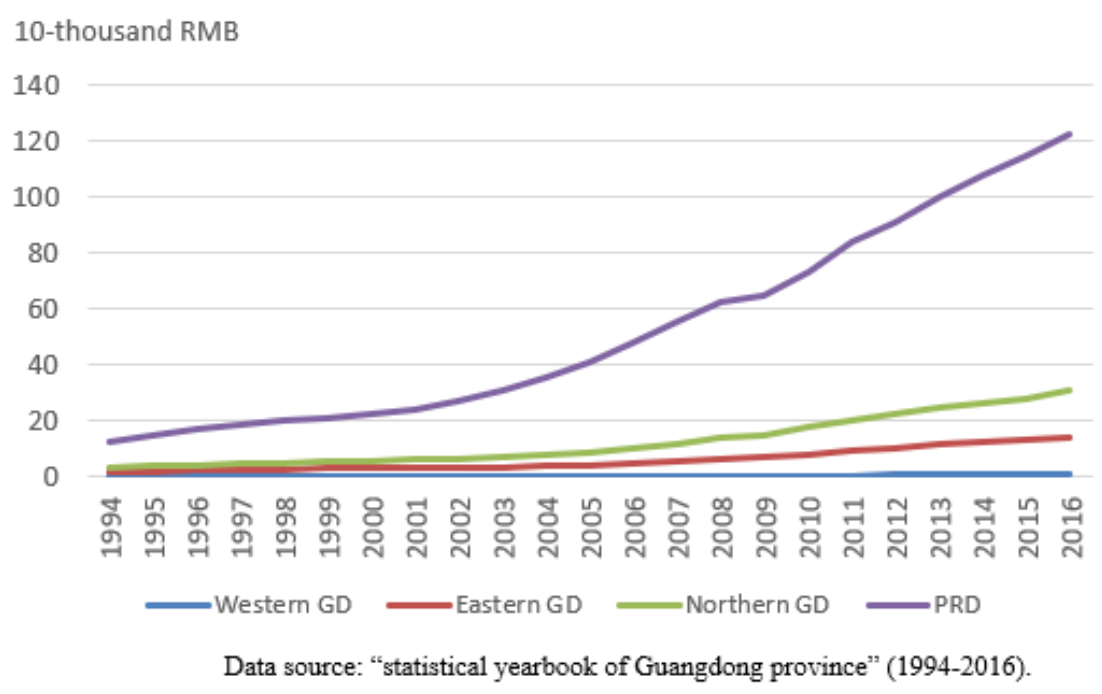

Figure 4. Growth process of per capita GDP in four regions of Guangdong from 1994 to 2016 Data source: "statistical yearbook of Guangdong province" (1994-2016).

\section{Analysis of Causes of Regional Economic Differences in Guangdong}

\subsection{Theoretical Analysis of Regional Economic Differences}

The classical theory of national economic accounting holds that national income is determined jointly by consumption, investment, government expenditure and import and export. Economists such as Keynes have verified the practicality of this theory. In recent years, domestic scholars have made relevant qualitative analysis on the causes of regional economic differences, but quantitative analysis is rare, and quantitative analysis combined with spatial effect is rare. Ren jianjun et al. [6] analysed the phenomena and causes of China's regional economic development differences, and found that China's regional economic differences have expanded continuously, influenced by factors such as fixed asset investment, income consumption capacity, foreign economic trade and fiscal policies. Li jianbao et al. [7] found that regional economic differences are significantly influenced by external policies, and introduced spatial measurement method to measure the externality and transmission mechanism of economic differences, and measured the direction and effect of externality on the basis of testing the spatial correlation.

\subsection{Spatial Correlation Test}

When analysing the per capita GDP data of Guangdong cities over the years, it is found that the per capita GDP data of Guangdong cities have obvious spatial correlation characteristics, that is, the economic data correlation between adjacent cities is high and significant. Moran's, I index is used to measure the spatial autocorrelation trend of regional economy in Guangdong over the years, and the results are shown in figure 5.

In recent 14 years, Moran's I index of regional economy in Guangdong has fluctuated to a certain extent, but it is above 0.45 every year and highly significant, indicating that regional economy in Guangdong has a strong spatial autocorrelation. From 2000 to 2016, the statistics of Moran's I of regional economy in Guangdong showed an upward trend, indicating that regional economic development in Guangdong has an increasingly strong spatial correlation, reflecting that urban economic development can effectively drive the coordinated development of surrounding cities, and there is a strong positive externality in space. 


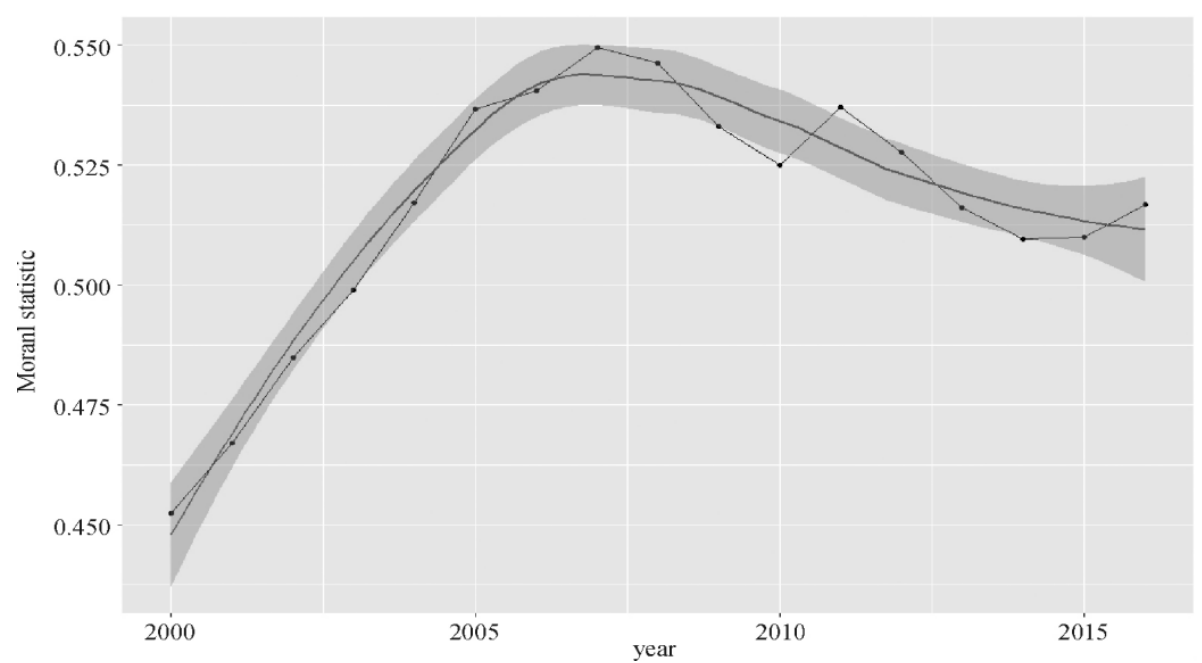

Figure 5. The annual trend of Moran's I values related to regional economy in Guangdong

\subsection{Spatial Error Model Test}

According to the classic analysis paradigm of national economic accounting in macroeconomics, and referring to the analysis methods of $\mathrm{Li}$ jianbao et al. [7] and Cao fangdong et al., the per capita regional GDP (GDP) is selected as the explained variable. At the same time, local fiscal expenditure per capita (GPE), social retail sales per capita (FCE), total import and export per capita (TEI) and total fixed asset investment per capita (IFA) are selected as explanatory variables. At the same time, considering the policy influences such as the "outline of the reform and development planning of the pearl river delta", the policy dummy variable (ZC) is introduced, and the spatial error model of panel data is constructed by using the relevant data of Guangdong cities from 2003 to 2016. To examine the causes and spatial effects of regional economic differences in Guangdong, the data are obtained from "Guangdong statistical yearbook" (2014-2017). Take the logarithm of all the data to eliminate the multicollinearity and heteroscedasticity, substitute it into the space lag model (2), and use the maximum likelihood method to estimate the measurement results of spatial panel data of individual fixed effects, as shown in table 1.

Table 1. Spatial measurement results of regional economic difference cause in Guangdong

\begin{tabular}{|c|c|c|c|c|}
\hline & Regression coefficient & Standard deviation & $\mathbf{t}$ & $\mathbf{p}$ \\
\hline GPE & 0.3561 & 0.0364 & 9.7838 & $0 * * *$ \\
\hline FCE & 0.3207 & 0.0255 & 12.5797 & $0 * * *$ \\
\hline TEI & 0.1042 & 0.0185 & 5.6440 & $0 * * *$ \\
\hline IFA & 0.0524 & 0.0310 & 1.6870 & 0.0916. \\
\hline ZC & 0.3334 & 0.0575 & 5.7935 & $0 * * *$ \\
\hline$\rho$ & 0.6589 & 0.0425 & 15.497 & $0 * * *$ \\
\hline
\end{tabular}

Note: "***", "**", "*" and ". "represent significant at levels of 0.001, 0.01, 0.05 and 0.1, respectively.

From the regression results of the spatial error model, it can be found that each explanatory variable is very significant. Local fiscal expenditure per capita (GPE) increases by $1 \%$, which can drive the growth of per capita GDP by $0.3561 \%$. Social retail sales per capita (FCE) can increase per capita GDP by $0.3207 \%$ for every $1 \%$ increase. Every $1 \%$ increase in total import and export per capita (TEI) can boost per capita GDP growth by $0.1042 \%$. Every $1 \%$ increase in total fixed asset investment per capita (IFA) can boost per capita GDP by $0.0524 \%$. It is worth noting that pearl river delta urban agglomeration development policy support is one of the main factors influencing regional economic differences in Guangdong. In addition, the spatial error coefficient $\rho$ is 0.6589 and is highly significant, indicating that regional economy in Guangdong has obvious spatial error effect, that is, urban 
economic growth will drive the coordinated development of surrounding cities through the diffusion effect.

\section{Research Conclusion}

Through exploratory spatial data analysis (figure 1), it is found that in the past 14 years, the economy in Guangdong cities has obvious spatial differences, and the regional economic evolution tends to be balanced, showing that the second and third tier cities with relatively developed economy have gradually increased, while the fourth-tier cities with relatively backward economy have significantly decreased.

The Gini coefficient, Theil index, variation coefficient and Herfindahl index of regional economy in Guangdong are comprehensively investigated, and it is found that the economic difference index shows an inverted U-shape trend (as shown in Figure 2), indicating that the economic difference in Guangdong has first increased and then decreased since 2003. The inflection point occurred around 2008. In recent years, the economic difference has a tendency of continuous decrease.

According to the traditional macroeconomic theory, economic policies, consumption, investment, government expenditure and import and export are all important factors affecting economic development. According to the panel data, the spatial error model is constructed, and the test results show that economic policy is one of the main factors affecting regional economic differences in Guangdong. At the same time, the regional economy of Guangdong has obvious positive externality, that is, the economic growth of a city will drive the economic growth of surrounding cities through the diffusion effect.

In the future, cities with strong economy in Guangdong can continue to maintain a good development momentum by innovation-driven industrial upgrading. The relatively backward regions can improve the competitiveness of urban economy by expanding consumption, increasing investment, expanding fiscal policy, developing international trade and policy support, and finally achieve the overall coordinated development of Guangdong regional economy.

\section{References}

[1]. Tan chenglin, Zheng yunfeng, Zhang hua. Analysis on the trend and characteristics of the coordinated development of China's regional economy [J]. Economic geography,2013(1):9-14.

[2]. Hu shaodong, Liang qiang, Xu zongling. Analysis of regional economic growth and difference in Guangdong province from 1992 to 2005 [J]. Southern economy,2007(4):74-82.

[3]. Wang shaojian, Wang yang, Zhao yabo. Study on multi-scale and multi-mechanism of regional economic difference in Guangdong province [J]. Geoscience,2014(10):1184-1192.

[4]. Wen chao. An empirical study on the coordinated development of Guangdong urban economy based on exploratory spatial data analysis [J]. Journal of Guangdong university of science and technology,2016(21):71-78.

[5]. Liu xiaoming, Wei yingqi, Li guoping. Convergence or divergence? Literature review on the debate on regional economic development in China [J]. Economic research,2004,39(7):70-81.

[6]. Ren jianjun, Yang guoliang. Analysis of regional economic development differences and causes in China and [J]. Economic geography,2010(5):784-789.

[7]. Li jianbao, Bai yongping, Li jianhu, et al. Analysis of regional economic differences based on spatial econometric model: taking the radiation belt of Lanzhou-Xinjiang railway as an example $[\mathrm{J}]$. Resources and environment in arid regions, 2012(10):144-149. 